\title{
OESOPHAGUS
}

\section{The Munich Barrett follow up study: suspicion of Barrett's oesophagus based on either endoscopy or histology only- what is the clinical significance?}

\author{
A Meining, R Ott, I Becker, S Hahn, J Mühlen, M Werner, H Höfler, M Classen, W Heldwein, \\ T Rösch
}

Background: The incidence of distal oesophageal adenocarcinoma is rising, with chronic reflux and Barrett's oesophagus being considered risk factors. Reliable detection of Barrett's oesophagus during upper endoscopy is therefore mandatory but requires both endoscopy and histology for confirmation. Appropriate management of patients with endoscopic suspicion but negative on histology, or vice versa, or of patients with no endoscopic suspicion but with a biopsy diagnosis of intestinal metaplasia at the gastro-oesophageal junction, has not yet been studied prospectively.

Patients and methods: In a prospective multicentre study, 929 patients (51\% male, mean age 50 years) referred for upper gastrointestinal endoscopy were included; $59 \%$ had reflux symptoms. The endoscopic aspect of the $Z$ line and any suspicion of Barrett's oesophagus were noted, and biopsies were taken in all patients from the $Z$ line $(n=4)$, gastric cardia $(n=2)$, and body and antrum $(n=2$ each). Biopsies positive for specialised intestinal metaplasia (SIM) were reviewed by a reference pathologist for a final Barrett's oesophagus diagnosis. All patients with endoscopic and/or histological suspicion of Barrett's oesophagus were invited for a follow up endoscopy; the remaining cases (no endoscopic or histological suspicion of Barrett's oesophagus) were followed clinically.

Results: Of 235 patients positive for Barrett's oesophagus on endoscopy and/or histology, $63 \%$ agreed to undergo repeat endoscopy (mean follow up period 30.5 months). $46 \%$ of patients with an endoscopic Barrett's oesophagus diagnosis but no histological confirmation (group A) showed the same distribution, a

See end of article for authors' affiliations

......

Correspondence to: Professor T Roesch, Central Interdisciplinary, Endoscopy Unit Department of Gastroenterology, Campus Virchow, Charité University Hospitals, Berlin, Germany; Thomas. Roesch@charite.de

Revised version received 22 March 2004

Accepted for publication 31 March 2004 further $42 \%$ did not have Barrett's oesophagus, and $11 \%$ had confirmed Barrett's oesophagus on both endoscopy and biopsy on follow up. In the group with a histological Barrett's oesophagus diagnosis but negative on initial endoscopy (group B), follow up showed the same in $26 \%$ whereas $46 \%$ had no Barrett's oesophagus, and confirmed Barrett's oesophagus (endoscopy plus histology) was diagnosed in 17\%. Of the study population, 16 patients had Barrett's oesophagus on initial endoscopy confirmed by histology which remained constant in $70 \%$ at follow up (group C). Of the remaining patients without an initial Barrett's oesophagus diagnosis on either endoscopy or histology (group D) and only clinical follow up (mean follow up period 38 months), one confirmed Barrett's oesophagus case was found among 100 patients re-endoscoped outside of the study protocol. However, no single case of dysplasia or cancer of the distal oesophagus was detected in any patient during the study period.

Conclusions: Even in a specialised gastroenterology setting, reproducibility of presumptive endoscopic or histological diagnoses of Barrett's oesophagus at follow up were poor. Only 10-20\% of cases with either endoscopic or histological suspicion of Barrett's oesophagus had established Barrett's oesophagus after 2.5 years of follow up. The risk of dysplasia in this population was very low and hence meticulous follow up may not be required.
$\mathrm{T}$ he increasing incidence of distal oesophageal adenocarcinoma, ${ }^{12}$ which is probably related to Barrett's oesophagus and chronic reflux disease, has focused attention on endoscopic detection of Barrett's oesophagus. Debate has arisen as to how to reliably diagnose Barrett's oesophagus in its various forms (long/short) on endoscopy and whether biopsies should be taken in small tongues of metaplasia, or even from the normal gastro-oesophageal junction on endoscopy, to identify short and incipient forms of Barrett's oesophagus. According to recent guidelines, ${ }^{3}$ Barrett's oesophagus is diagnosed on endoscopy and confirmed on histopathology by the presence of specialised intestinal metaplasia (SIM), and follow up is recommended in both short and long forms although there has been much debate on this topic. ${ }^{4}$

As the clinical consequences of a diagnosis of Barrett's oesophagus is lifelong follow up, precise definition of patients who fall into the Barrett's oesophagus category is mandatory.
The question of whether a patient diagnosed as having Barrett's oesophagus on endoscopy but without histological confirmation, or vice versa (a patient with a biopsy diagnosis of Barrett's oesophagus at an endoscopically inconspicuous gastro-oesophageal junction), requires increased vigilance, remains to be answered. Intestinal metaplasia at a normal gastro-oesophageal junction has been found in 6-36\% of patients endoscoped for various reasons, ${ }^{56}$ but follow up data are limited. On the other hand, patients with an endoscopic impression of Barrett's oesophagus but unconfirmed on biopsy have not been examined.

We therefore carried out a prospective study involving a large number of unselected patients within the framework of a multicentre (in- and outpatient) endoscopy setting, with the primary study aim of evaluating the medium term course of patients with either endoscopic or histological suspicion of

Abbreviations: SIM, specialised intestinal metaplasia 
Barrett's oesophagus, without initial confirmation by the respective other test.

\section{PATIENTS AND METHODS \\ Selection of patients}

This multicentre study was conducted by the Munich Gastroenterology Working Group. Patients were recruited during an eight month period at eight gastroenterological centres. Any patient referred for upper gastrointestinal endoscopy for evaluation of dyspepsia and/or reflux symptoms was eligible for inclusion if aged between 18 and 80 years with no previous history of Barrett's oesophagus or dysplasia/cancer of the upper gastrointestinal tract. Further exclusion criteria were a known malignant disease, previous (partial or complete) gastrectomy, severe comorbidity, unwillingness to participate or inability to provide informed consent, and conditions that precluded safe biopsy (coagulation disorders, portal hypertension with oesophageal varices). To avoid selection bias, the first three eligible patients per day were included at the participating centres during various time periods, according to the availability of research assistants.

\section{Endoscopy}

Endoscopy was performed after an overnight fast; 18 endoscopists, all with extensive experience (at least three years' experience of performing upper gastrointestinal endoscopy and more than 3000 examinations) participated in the study. All examinations were conducted according to a strict protocol, with the examiner dictating the findings to the research assistant during the following examinations: location of the $\mathrm{Z}$ line (change of pale-whitish squamous epithelium to red columnar epithelium); upper end of the proximal gastric folds; and passage through the diaphragmatic hiatus. Endoscopic distances were measured in centimetres from the incisors. The extent of columnar epithelium in the distal oesophagus was defined as the distance between the proximal gastric folds on moderate air insufflation and the most proximal extension of columnar epithelium ( $\mathrm{Z}$ line), giving rise to a suspected diagnosis of Barrett's oesophagus on endoscopy (short Barrett's $<3 \mathrm{~cm}$, long Barrett's $\geqslant 3 \mathrm{~cm}$ ). In addition, the presence or absence of a hiatal hernia was noted. If oesophagitis was found, the endoscopist graded its severity in accordance with the Savary-Miller classification. These definitions were agreed in a consensus discussion between participants (including endoscopists and histopathologists) before the start of the study.

Two biopsies from the antrum, gastric body, and cardia ( $2 \mathrm{~cm}$ below the proximal end of the gastric folds, at the 12 , 3,6 , and 9 o'clock positions with the patient in the left lateral position) were obtained in all patients, as were a further four biopsies from just below the $\mathrm{Z}$ line. In addition, if Barrett's oesophagus was suspected, four quadrant biopsies of the columnar epithelium (including tongues) were taken at intervals of $2 \mathrm{~cm}$.

\section{Histopathology}

Biopsy specimens were fixed in $4 \%$ buffered formalin. Slides were stained using a combination of haematoxylin-eosin and Alcian blue at $\mathrm{pH} 2.5$. Any gastritis in the antrum or gastric body was graded and classified in accordance with the updated Sydney system. ${ }^{7}$ Histological evidence of SIM at the oesophagogastric junction was defined as columnar-type epithelium with goblet cells staining positive with Alcian blue.

All histological examinations were carried out by a total of five experienced gastrointestinal pathologists (see appendix) after agreement on criteria and definitions. In addition, both the initial and follow up biopsy specimens from patients who were selected for follow up (groups A-C, see below) were reviewed a second time by an experienced reference pathologist (IB) who had no knowledge of the clinical history, results of endoscopy, or previous histological findings.

\section{Questionnaire}

On the same day of the primary and follow up endoscopy examinations, all patients were asked to complete a questionnaire on symptoms, including heartburn, regurgitation, and dysphagia. Frequency and dosage of antacids, $\mathrm{H}_{2}$ blockers, and proton pump inhibitors were also noted.

\section{Selection of patients for follow up Endoscopic follow up}

Patients with any suspicion of Barrett's oesophagus on endoscopy and/or histology were invited to attend follow up examinations (at least 1.5 years after the initial examination) at the same institution at which the index endoscopy was carried out:

- endoscopically visible extension of the columnar epithelium into the lower oesophagus (that is, endoscopically suspected Barrett's oesophagus) without histological confirmation of SIM in any of the biopsies (at least four) obtained from this area (group A: endo pos, histo neg);

- histological detection of SIM at the Z line without endoscopically visible extension of the columnar epithelium into the lower oesophagus (group B: endo neg, histo pos);

- endoscopically diagnosed and histologically confirmed Barrett's oesophagus (group C).

At the follow up examination, clinical, endoscopic, and histological examinations were conducted in the same way as during the initial endoscopies, including biopsies. With respect to endoscopy, all investigators were aware of the results of the initial examinations.

\section{Clinical follow up}

The remaining patients (group D: no Barrett's oesophagus on endoscopy or biopsy) were contacted by telephone to determine whether they had suffered from cancer of the oesophagus. In addition, patients were asked whether a subsequent endoscopy had been performed. If this was the case, the respective physician was contacted and endoscopy and histological files were screened for endoscopic or histological presence of Barrett's oesophagus or dysplasia.

\section{Statistical analysis}

Statistical analysis of unpaired data was carried out using the $\chi^{2}$ test or Fisher's exact test. The Wilcoxon signed rank test for paired samples was used to check for statistically significant differences between findings obtained during the initial and follow up examinations. A $\kappa$ statistic was

\begin{tabular}{|c|c|}
\hline \multicolumn{2}{|l|}{ Clinical data } \\
\hline $\operatorname{Sex}(M / F)$ & $51.3 \% / 48.7 \%$ \\
\hline Age (y) (mean (SD)) & $50.1(13.6)$ \\
\hline Heartburn at least once a week: & $58.8 \%$ \\
\hline Use of proton pump inhibitors: & $24.4 \%$ \\
\hline \multicolumn{2}{|l|}{ Endoscopic findings } \\
\hline Hiatal hernia & $39.2 \%$ \\
\hline Reflux oesophagitis & $19.7 \%$ \\
\hline Suspicion of Barrett's oesophagus (long/short) & $11.8 \%(2.0 \% / 9.8 \%)$ \\
\hline \multicolumn{2}{|l|}{ Histopathological findings } \\
\hline Intestinal metaplasia at the OGJ & $16.3 \%$ \\
\hline Helicobacter pylori associated gastritis & $33.4 \%$ \\
\hline Intestinal metaplasia in the stomach & $15.0 \%$ \\
\hline
\end{tabular}




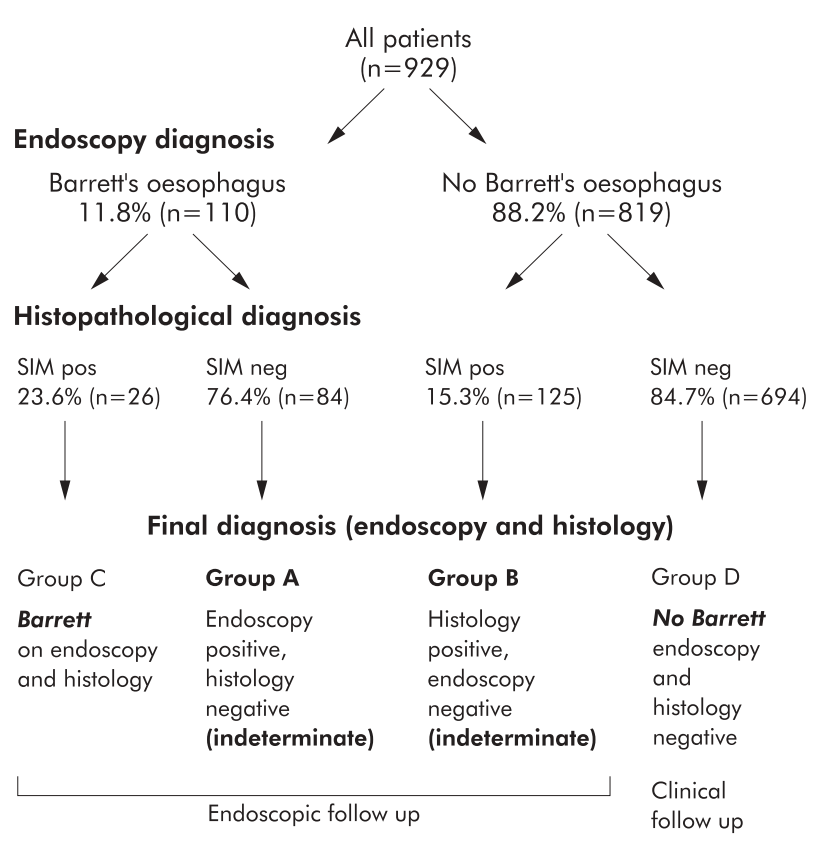

Figure 1 Overview of all study patients and their endoscopic and histopathological results concerning a diagnosis of Barrett's oesophagus and the presence of specialised intestinal metaplasia (SIM) at the oesophagogastric junction.

calculated to estimate the agreement between the "standard" pathologist and the blinded reference pathologist with respect to finding of SIM. A $\kappa$ value $<0.4$ was considered to show no agreement, fair agreement was represented by $\kappa=$ 0.4-0.75, and excellent agreement was shown by $\kappa=$ 0.75-1.0. In addition, multivariate logistic regression analysis was carried out to identify independent markers in the patient history, histological findings, or endoscopic findings for the presence of SIM at the initial examination, as well as for the consistency of the initial findings for the three groups at follow up examinations. A p value of less than 0.05 was considered statistically significant. The Statistical Package for the Social Sciences (SPSS) for Windows program, version 10.1, was used (SPSS Inc., Chicago, Illinois, USA).

\section{Ethics}

The study was approved by the ethics committee of the Technical University of Munich. Patients were informed that the main aim of the study was to clarify the course (risk of cancer) of an endoscopic and/or histological diagnosis of Barrett's oesophagus.

\section{RESULTS}

\section{Patient characteristics}

Among 1014 dyspeptic patients recruited to the study, a thorough patient history and histological and endoscopic

Table 2 Clinical and patient history parameters significantly associated with intestinal metaplasia at the oesophagogastric junction (according to multiple logistic regression analysis)

\begin{tabular}{llll}
\hline Parameter & Odds ratio & $\mathbf{9 5 \%} \mathrm{Cl}$ & $\mathbf{p ~ V a l u e ~}$ \\
\hline Gastric intestinal metaplasia & 1.716 & $1.013-2.906$ & 0.045 \\
Age over 50 y & 1.642 & $1.058-2.549$ & 0.027 \\
Male sex & 1.524 & $1.008-2.304$ & 0.046 \\
\hline $\mathrm{Cl}$, confidence interval. & & & \\
\hline
\end{tabular}

work up, as specified by the study protocol, was available for 929 patients, with the remaining patients being excluded for protocol violations (refusal to answer the questionnaire, endoscopists not taking biopsies or taking insufficient biopsies, or missing relevant descriptions, etc). Clinical data and endoscopic and histopathological findings for the 929 study patients are shown in table 1 .

\section{Endoscopic and histopathological findings at the index endoscopy}

Based on the endoscopic and histopathological diagnoses, patients were classified into four groups (fig 1).

\section{Indeterminate Barrett's oesophagus cases}

- Group A ("endoscopic Barrett"): endoscopic diagnosis of Barrett's oesophagus but no histopathological confirmation ( $\mathrm{n}=84$; mean age 53.0 (12.5) years). Endoscopic suspicion was long Barrett's oesophagus $(\mathrm{n}=10)$ and short Barrett's oesophagus $(\mathrm{n}=74)$.

- Group B ("histological Barrett"): endoscopically normal distal oesophagus and $\mathrm{Z}$ line but histopathological diagnosis of SIM on four quadrant biopsies, indicating possible Barrett's oesophagus $(\mathrm{n}=125$; mean age 54.1 ( 11.5 ) years).

\section{Cases with definite Barrett's oesophagus diagnosis}

- Group C (Barrett): endoscopic diagnosis of Barrett's oesophagus, confirmed by histopathology $(\mathrm{n}=26$; mean age 55.1 (12.5) years; nine with long and 17 with short Barrett's oesophagus).

- Group D (no Barrett): patients without endoscopic or histopathological evidence of Barrett's oesophagus; these were the remaining 694 cases (mean age 48.8 (13.9) years).

Barrett's oesophagus was diagnosed by endoscopy, according to predefined criteria, in 110 patients $(n=91$ short Barrett's oesophagus; $\mathrm{n}=19$ long Barrett's oesophagus). Histopathology confirmed Barrett's oesophagus by the presence of SIM in 26 of these 110 patients $(23.6 \%)$ compared with 125 of 819 patients (15.3\%) with no visible endoscopic extension of columnar epithelium into the distal oesophagus on endoscopy ( $\chi^{2}$ test, $\mathrm{p}=0.025$ ).

A multivariate regression analysis was carried out on clinical data (age $\leqslant 50$ or $>50$ years, sex, presence or absence of heartburn, use of proton pump inhibitors), as well as on endoscopic findings (presence of absence of hiatal hernias, reflux oesophagitis, and endoscopic suspicion of Barrett's oesophagus) and histological findings (presence or absence of intestinal metaplasia in the stomach and Helicobacter pylori infection). This analysis revealed that the presence of intestinal metaplasia elsewhere in the stomach, age over 50 years, and male sex were parameters significantly associated with histological detection of SIM (all groups). The respective odds ratios and significance levels are shown in table 2 .

\section{Follow up results}

Endoscopic follow up was offered to all patients in groups $\mathrm{A}-\mathrm{C}(\mathrm{n}=235)$ and $63 \%(\mathrm{n}=148 ; 53 \%$ men, $47 \%$ women; mean age 55.8 (10.6) years) agreed to be re-endoscoped. Repeat endoscopies were performed over a 24 month period, at least 18 months after the index endoscopy in every patient (mean 30.54 (range 6.14) months after the index endoscopy). Follow up was not possible in 87 patients-mostly contact lost due to moving house, unwillingness to undergo a further endoscopy, death due to unrelated causes $(n=8)$, or severe comorbidity $(\mathrm{n}=3)$. There were no differences 


\begin{tabular}{|c|c|c|}
\hline Initial result & Follow up results & $\%$ \\
\hline Group A & Same diagnosis & $46 \%$ \\
\hline $\begin{array}{l}\text { Endoscopy } \\
\text { positive, } \\
\text { histology } \\
\text { negative } \\
\text { (indeterminate) } \\
\mathrm{n}=49\end{array}$ & $\begin{array}{l}\text { Endoscopy pos, histology pos (Barrett) } \\
\text { Endoscopy neg, histology pos (group B) } \\
\text { Endoscopy neg, histology neg (no Barrett) }\end{array}$ & $\begin{array}{l}11 \% \\
0 \\
42 \%\end{array}$ \\
\hline Group B & Same diagnosis & $26 \%$ \\
\hline $\begin{array}{l}\text { Histology } \\
\text { positive, } \\
\text { endoscopy } \\
\text { negative } \\
\text { (indeterminate) } \\
n=83\end{array}$ & $\begin{array}{l}\text { Endoscopy pos, histology pos (Barrett) } \\
\text { Endoscopy pos, histology neg (group A) } \\
\text { Endoscopy neg, histology neg (no Barrett) }\end{array}$ & $\begin{array}{l}17 \% \\
11 \%\end{array}$ \\
\hline $\begin{array}{l}\text { Group C } \\
\text { Barrett } \\
\text { on endoscopy } \\
\text { and histology } \\
\mathrm{n}=16\end{array}$ & $\begin{array}{l}\text { Same diagnosis } \\
\text { Endoscopy pos, histology neg (group A) } \\
\text { Endoscopy neg, histology pos (group B) } \\
\text { Endoscopy neg, histology neg (no Barrett) }\end{array}$ & $\begin{array}{l}15 \% \\
0\end{array}$ \\
\hline $\begin{array}{l}\text { Group D } \\
\text { No Barrett } \\
\text { on endoscopy } \\
\text { and } \\
\text { histology } \\
\mathrm{n}=100\end{array}$ & $\begin{array}{l}\text { Same diagnosis } \\
\text { Endoscopy pos, histology pos (Barrett) } \\
\text { Endoscopy pos, histology neg (group A) } \\
\text { Endoscopy neg, histology pos (group B) }\end{array}$ & $9 \%$ \\
\hline
\end{tabular}

Figure 2 Overview of endoscopic and clinical follow up results of all patients concerning a diagnosis of Barrett's oesophagus and the presence of specialised intestinal metaplasia at the oesophagogastric junction. Biopsies from the normal appearing $Z$ line were obtained in only nine of 100 re-endoscoped patients in group $\mathrm{D}$.

between those attending follow up and those lost to follow up with respect to age or sex. In addition, there was little difference in relation to endoscopic-histological diagnoses (follow up in groups $\mathrm{A} / \mathrm{B} / \mathrm{C}$ : $65 \% / 66 \% / 62 \%$ ).

Of the 694 patients in group D with an index endoscopy and histology negative for Barrett's oesophagus, 406 were contacted by telephone (58.5\%).

Follow up results in indeterminate Barrett's oesophagus cases: groups A (endoscopy positive) and $B$ (histology positive)

Endoscopic and histological findings at the initial and follow up investigations are shown in fig 2 .

- In group A ( $\mathrm{n}=49$; “endoscopic Barrett's oesophagus": index endoscopy positive for Barrett's oesophagus but histology negative), the same diagnosis was obtained in $46 \%$ of cases; endoscopic suspicion was not maintained in $42 \%$ whereas Barrett's oesophagus diagnosed endoscopically at the index endoscopy was finally confirmed by histology on the follow up endoscopy in approximately $10 \%$ of case. These included one patient with an endoscopic diagnosis of long segment Barrett's oesophagus and five of 48 patients with an endoscopic diagnosis of short segment Barrett's oesophagus.

- In group B ( $\mathrm{n}=83$; "histological Barrett's oesophagus": index endoscopy negative, histology positive for SIM), the diagnosis remained the same in approximately $25 \%$ of cases but in almost half of the cases SIM was no longer detected on biopsies. A new endoscopic suspicion of Barrett's oesophagus was raised at the follow up endoscopy in $28 \%$ (all of these cases were classified as short
Barrett's oesophagus), and histological confirmation was obtained in $61 \%$ of these endoscopy positive cases. A total of $17 \%$ therefore had endoscopically and histologically confirmed short Barrett's oesophagus that had not previously been recognised at the index endoscopy.

Follow up results in definite Barrett's oesophagus diagnoses: groups $C$ (Barrett) and $D$ (no Barrett)

- In group C ( $n=16$; Barrett-that is, index endoscopy and histology positive for Barrett's oesophagus), the diagnosis remained the same on follow up in $70 \%$ of cases, the remaining cases becoming indeterminate. In relation to the endoscopic diagnosis of long or short Barrett's oesophagus, Barrett's oesophagus was histologically confirmed in all four patients with a long segment and in seven of 12 patients with short segment Barrett's oesophagus.

- Group D was primarily followed clinically, and complete follow up information was obtained in 406 cases. None of the patients had undergone an operation or been treated for carcinoma of the distal oesophagus. A further endoscopy (although this was not part of the study protocol) was performed in 100 of 406 cases (24.6\%) after a mean follow up period of 38 months (range 2446 months). Review of the endoscopic and histological results in these patients showed that Barrett's oesophagus was described endoscopically in 10 cases $(10 \%)$, which was confirmed by histology in a single case $(1 \%)$. In one further case, no biopsies were obtained from the suspected Barrett's oesophagus area (reasons unknown). In nine patients, biopsies were taken from the $\mathrm{Z}$ line although there was no endoscopic suspicion of Barrett's oesophagus. Histology revealed SIM in one of these nine patients (fig 2).

\section{Detection of dysplasia during the study}

Dysplasia or intraepithelial neoplasia was not found histologically either at the index endoscopy or at follow up endoscopy in any patient in any group.

\section{Reproducibility on follow up}

Reproducibility of the endoscopic and histological findings at the index endoscopy was heterogeneous, as shown above (see fig 2). This inconsistency between the initial findings and findings obtained at follow up examinations proved to be highly significant (Wilcoxon test, $\mathrm{p}<0.001$ ). Combining groups $\mathrm{A}-\mathrm{C}$, initial findings (endoscopy plus histology) were confirmed in only $37.2 \%$ of cases. Clinical data such as presence of reflux symptoms, use of proton pump inhibitors, age, and sex were not significantly associated with the divergent results obtained at the follow up examinations (multiple logistic regression analysis, all $\mathrm{p}>0.1$ ).

\section{Consistency of histopathological diagnosis}

All cases of positive findings either on endoscopy (suspicion of Barrett's oesophagus) or histopathology (positive for SIM) from groups A-C were reviewed by the reference pathologist. With regard to the diagnosis of SIM, there was disagreement between the initial gastrointestinal pathologist and the reference pathologist in $18.9 \%$ of cases (data for initial and follow up examinations combined). However, the respective $\kappa$ values improved from 0.443 during the initial study examination (biopsies during the index endoscopy) to 0.712 at the follow up examination $(p<0.001)$. The main reason for this discrepancy was misdiagnosis of Alcian blue positive findings in the foveolar epithelium for goblet cell metaplasia (95.4\% of all inconsistent findings). 


\section{DISCUSSION}

The present study attempted to analyse the relevance of an indeterminate diagnosis of Barrett oesophagus on either endoscopy or histology. This was done by performing endoscopic and clinical follow up for more than 2 years. Several conclusions can be drawn from the results of our study.

- The consistency of the endoscopic and histological findings between the index and follow up endoscopies was poor in this multicentre setting, and in only a third of cases were the same results obtained. This was despite participation of experienced gastroenterological endoscopists.

- In cases with indeterminate diagnoses (endoscopy or biopsy positive), confirmed Barrett's oesophagus diagnosed on endoscopy and histology was found in 10-20\% of cases on follow up endoscopies. No Barrett was found in $40-50 \%$ of cases on follow up.

- Confirmed Barrett's oesophagus remained so on follow up endoscopy in only $70 \%$ of cases. Moreover, of the majority of patients who initially had no evidence of Barrett's oesophagus on endoscopy and histology, Barrett's oesophagus was diagnosed or suspected at other institutions outside of the study protocol in up to $10 \%$ of those patients re-endoscoped.

- Despite the disappointing results of endoscopy and histology, the risk of developing dysplasia in our patients appeared to be very low and was 0 in our study after a mean follow up of more than 30 months in all groups.

Therefore, our study indicates that "screening" for Barrett's oesophagus in patients undergoing routine endoscopy by means of non-selective biopsy of the $\mathrm{Z}$ line is not worthwhile for a variety of reasons. Notably, these results were not different for patients examined for reflux symptoms and for those without these complaints.

Our results can be interpreted in two further ways. Firstly, regression or loss of SIM or short Barrett could be the reason for the divergent results, possibly due to other factors, such as the use of proton pump inhibitors. ${ }^{8}$ However, in the present study neither progression nor regression of the initial findings was associated with the use of proton pump inhibitors. It therefore appears likely that the heterogeneous course of the endoscopic findings is related more to an inability to describe columnar epithelium spreading into the lower oesophagus accurately by endoscopic means or possible sampling errors on endoscopic biopsy for SIM. SIM is often distributed focally so that there is potential bias even when multiple biopsies are taken, as in the present study. In addition, the gastro-oesophageal junction is prone to motion artefacts caused by the patient breathing and retching. In another study from our group, complete documentation of the entire $\mathrm{Z}$ line on still images or video sequences was possible in only about $25 \%$ of cases. ${ }^{9}$ Therefore, we conclude from these results that the methods currently used to detect precancerous conditions at the oesophagogastric junction need to be improved. Staining techniques using methylene blue $^{10-12}$ and magnification endoscopy ${ }^{13}$ have been evaluated and have provided good results in smaller single centre studies. However, many of these methods are time consuming and expensive. In addition, the data available regarding these new methods are still rather variable ${ }^{14}$ and therefore can only be considered as preliminary until larger studies comparing the accuracy of different methods become available.

There are several potential limitations of our study. Patient selection was not based on reflux symptoms but more than two thirds of our patients reported such symptoms. Our results did not differ in these two groups and we deliberately decided not to limit this study to reflux patients. Furthermore, a multicentre setting with several endoscopists and histopathologists participating will always reveal less consistent results than in single centre trials with one or only few dedicated examiners. However, all participating endoscopists were experienced, and the criteria for endoscopic diagnosis of the $\mathrm{Z}$ line and potential Barrett's oesophagus were defined in consensus before the start of the study. We are furthermore convinced that this study better reflects reality than single centre studies with their inherent bias in terms of scientific interest or referral patterns. For the same reason, we did not blind examiners who performed the follow up examinations of the initial grouping of the patients; if we did this, reproducibility might have been even lower. With respect to histopathology as a potential pitfall of the study, variability of the different pathologists involved was controlled by review of all positive cases (group A-C) before and during the study. Hence, in summary, we suggest that based on the results of speciality centres, the risk of Barrett's oesophagus in reflux and the risk of developing malignancy in Barrett's oesophagus has been overestimated, as has been reported previously. ${ }^{415}$

Some of our results for the index endoscopy were in accordance with previous studies. Although in our study the rate of histologically proven Barrett's oesophagus was slightly higher in patients with an endoscopic description of long Barrett's oesophagus $(30.6 \% \vee 20.9 \%$ in short Barrett's oesophagus), a higher frequency of histologically proven Barrett's might be expected when using a systematic biopsy protocol, as in the present study. In the literature, histological confirmation of Barrett's oesophagus (long and short) in cases diagnosed on endoscopy to represent Barrett's oesophagus range from $20 \%$ to $50 \% .^{5616} 17$ The present results, summarising data from a large number of patients, are thus at the lower end of the range previously reported.

The frequency of intestinal metaplasia at the oesophagogastric junction in patients without an endoscopic suspicion of Barrett's oesophagus was $15.3 \%$ in our study. These results and the correlations identified in our patient population are in line with those of previous studies. ${ }^{16}{ }^{17}$ Other factors potentially associated with the presence of SIM in other studies, ${ }^{18-20}$ such as infection with $H$ pylori, or endoscopic or clinical reflux parameters, were not identified as risk factors in the present study. Interestingly, intestinal metaplasia elsewhere in the stomach was found to have the highest odds ratio for the presence of intestinal metaplasia at the $\mathrm{Z}$ line among all significant independent risk factors identified in the single variance test. This is in agreement with the studies of Trudgill and colleagues ${ }^{21}$ and Goldblum and colleagues. ${ }^{19}$ It has been suggested that SIM, if it is detected in biopsies taken from a normal appearing gastro-oesophageal junction, is caused by similar pathological mechanisms to those involved in intestinal metaplasia elsewhere in the stomach (that is, $H$ pylori infection in the majority of cases). ${ }^{20} 22$ However, when a multiple logistic regression analysis was carried out for each group separately (presence or absence of suspicion of endoscopic Barrett's oesophagus), gastric intestinal metaplasia was no longer found to be a significant independent risk factor. In addition, it was found that there were no differences with regard to the frequency of infection with $H$ pylori between the two groups. Thus although it seems fairly logical that junctional SIM represents different entities in patients with and without endoscopic signs of Barrett's oesophagus, this hypothesis was not supported by the data of the present study.

Follow up data on patients with SIM at the oesophagogastric junction are sparse and this study is the first systematic, prospective, large, follow up study in this setting. 
In our study, the rate of dysplasia in newly diagnosed SIM in a normal Z line as well as in all other patient groups was zero; in the much smaller studies of Sharma and colleagues ${ }^{23}$ and Goldstein, ${ }^{24}$ one of 119 pooled cases of SIM developed low grade dysplasia at follow up. In our view, the risk of cancer on the basis of SIM alone (that is, without endoscopically visible signs of Barrett's oesophagus) and even in the short form of Barrett's oesophagus appears to be very low, at least within a few years. This does not exclude a higher risk on longer follow up.

With respect to histopathology as a potential pitfall of the study, variability of the different pathologists involved was controlled by review of all positive cases (group A-C) before and during the study. Although the initial interobserver variation was only fair (at initial and follow up examinations), variability decreased (with the kappa value increasing from 0.4 to 0.7 ), possibly due to a learning curve effect.

According to our data, only definite Barrett's oesophagus appears to be reasonably reproducible $(70 \%)$. Nevertheless, our results raise several questions and concerns regarding today's practice of screening for Barrett's oesophagus. ${ }^{3}$ If the American College of Gastroenterology guidelines were applied in the present study population, patients who did not initially fulfil the endoscopic and histological criteria for Barrett's oesophagus, but who had endoscopically described and histologically proven short Barrett's oesophagus at the follow up examination ( $17 \%$ in our series), would not have been included in surveillance programmes. On the other hand, how should one manage patients such as those who had histologically proven Barrett's oesophagus at the initial endoscopy but in whom this was not confirmed either endoscopically or histologically $(30 \%)$ at the follow up examination? Should these patients be excluded from further surveillance? There are as yet no definite answers to these questions although the situation may reflect everyday practice. Perhaps the methods currently available for detecting Barrett's oesophagus are not satisfactory enough in every situation to allow the solutions proposed by experts in guidelines. There is considerable bias caused by sampling errors and the endoscopist's inability to detect precisely the presence and extent of Barrett's oesophagus.

Apart from improving our technical methods (endoscopic and histological) of reliably detecting Barrett's oesophagus and/or dysplasia at the oesophagogastric junction, future studies should also concentrate on alternative epidemiological and biological markers that may be able to identify patients with an increased cancer risk. It has recently been shown by Corley et al that less than $4 \%$ of Barrett's adenocarcinomas are detected during surveillance. ${ }^{25}$ In view of the rising cancer incidence rates $^{12}$ on the one hand, and the problems associated with surveillance programmes on the other, there is a need for better diagnostic criteria for detecting Barrett's oesophagus, particularly in its early forms.

\section{Authors' affiliations \\ A Meining, R Ott, S Hahn, J Mühlen, M Classen, T Rösch, Department of Medicine II, Klinikum rechts der Isar, Technical University of Munich, Germany \\ I Becker, M Werner, H Höfler, Department of Pathology, Technical University of Munich, Germany \\ W Heldwein, Department of Medicine, Klinikum Innenstadt, University of Munich, Germany}

\section{APPENDIX}

In addition to the authors, the following colleagues were actively involved in this study: University of Munich: M. Götzberger, A Eigler (Department of Gastroenterology),
B Wiebecke (Department of Pathology); Technical University of Munich: J Reichenberger, K Egger, B Neu, P Born, E Frimberger, HD Allescher (Department of Gastroenterology); Neuperlach Hospital, Munich: W Schmitt, J Gospos (Department of Gastroenterology), J Pitzl (Department of Pathology); Bogenhausen Hospital, Munich: W Schepp, C Zillinger (Department of Gastroenterology), E Keiditzsch (Department of Pathology); gastroenterologists in private practice: J Weingart, W Höchter, B Birkner, M Strauch, M Henke, P Janetschek; pathologist in private practice: B Kaduk.

\section{REFERENCES}

1 Pera M, Cameron AJ, Trastek VF, et al. Increasing incidence of adenocarcinoma of the esophagus and esophagogastric junction. Gastroenterology 1993; 104:510-13.

2 Devesa SS, Blot WJ, Fraumeni JF. Changing patterns in the incidence of esophageal and gastric carcinoma in the United States. Cancer 1998;83:2049-53.

3 Sampliner RE, and the Practice Parameters Committee of the American College of Gastroenterology. Updated practice guidelines on the diagnosis, surveillance, and therapy of Barrett's esophagus. Am J Gastroenterol 2002;97:1888-95.

4 Provencale D, Schmitt C, Wong JB. Barrett's esophagus: a new look at surveillance based on emerging estimates of cancer risk. Am J Gastroenterol 1999:94:2043-53.

5 Spechler SJ, Zeroogian JM, Antonioli DA, et al. Prevalence of metaplasia at the gastro-oesophageal junction. Lancet 1994;344:1533-6.

6 Weinstein WM, Ippoliti AF. The diagnosis of Barrett's esophagus: Goblets, goblets, goblets. Gastrointest Endosc 1996;44:91-4.

7 Dixon MF, Genta RM, Yardley JH, et al. Classification and grading of gastritis: the updated Sydney system. Am J Surg Pathol 1996;20:1161-81.

8 Sampliner RE. Reduction of acid exposure and regression of Barrett's esophagus. Dig Dis 2000;18:203-7.

9 Götzberger M, Klose J, Lewerenz B, et al. Bilddokumentation in der gastroenterologischen Endoskopie-Vergleich von Standbild- und Videodokumentation des gastroösophagealen Übergangs. Z Gastroenterol 2002;40:694(abstract).

10 Canto MI, Setrakian S, Willis JE, et al. Methylene blue staining of dysplastic and nondysplastic Barrett's esophagus: an in vivo and ex vivo study. Endoscopy 2001;33:391-400.

11 Sharma P, Topalovski M, Mayo MS, et al. Methylene blue chromoendoscopy for detection of short segment Barrett's esophagus. Gastrointest Endosc 2001;54:289-93.

12 Canto MI, Yoshida T, Gossner L. Chromoendoscopy of intestinal metaplasia in Barrett's esophagus. Endoscopy 2002;34:330-6.

13 Guelrud M, Herrera I, Essenfeld $H$, et al. Enhanced magnification endoscopy: a new technique to identify specialized intestinal metaplasia in Barrett's esophagus. Gastrointest Endosc 2001;53:559-65.

14 Meining A, Rösch T, Kiesslich R, et al. Inter- and intra-observer variability of magnification chromoendoscopy for detecting specialized intestinal metaplasia at the gastroesophageal junction. Endoscopy 2004;36:160-4.

15 Shaheen NJ, Crosby MA, Bozymski EM, et al. Is there publication bias in the reporting of cancer risk in Barrett's esophagus? Gastroenterology 2000; 1 19:333-8

16 Padda S, Ramirez FC. Accuracy in the diagnosis of short-segment Barrett's esophagus: the role of endoscopic experience. Gastrointest Endosc 2001;54:605-8.

17 Johnston MH, Hammond AS, Laskin W, et al. The prevalence and clinical characteristics of short segments of specialized intestinal metaplasia in the distal esophagus on routine endoscopy. Am J Gastroenterol 1996;91:1507-11.

18 Öberg S, Peters JH, DeMeester T, et al. Inflammation and specialized intestinal metaplasia of cardiac mucosa is a manifestation of gastroesophageal reflux disease. Ann Surg 1997;226:522-32.

19 Goldblum JR, Vicari JJ, Falk GW, et al. Inflammation and intestinal metaplasia of the gastric cardia: the role of gastroesophageal reflux and $\mathrm{H}$. pylori infection. Gastroenterology 1998;114:633-9.

20 Goldblum JR, Richter JE, Vaezi M, et al. Helicobacter pylori infection, not gastroesophageal reflux, is the major cause of inflammation and intestinal metaplasia of the gastric cardia. Am J Gastroenterol 2002;97:302-1 1.

21 Trudgill NJ, Suvarna SK, Kapur KC, et al. Intestinal metaplasia at the squamocolumnar junction in patients attending for diagnostic gastroscopy. Gut 1997;41:585-9.

22 Van Sandick JW, van Lanschot JB, van Felius L, et al. Intestinal metaplasia of the esophagus or esophagogastric junction. Am J Clin Pathol 2002;117:117-25.

23 Sharma P, Weston AP, Morales T, et al. Relative risk of dysplasia for patients with intestinal metaplasia in the distal oesophagus and in the gastric cardia. Gut 2000;46:9-13.

24 Goldstein NS. Gastric cardia intestinal metaplasia: biopsy follow-up of 85 patients. Mod Pathol 2000;13:1072-9.

25 Corley DA, Levin TR, Habel LA, et al. Surveillance and survival in Barrett's adenocarcinomas: a population-based study. Gastroenterol 2002; 122:633-40. 